\title{
Diffuse Interstellar Bands: The Way Forward
}

\author{
A. G. G. M. Tielens \\ Leiden Observatory, Leiden University, PO Box 9513, NL-2300RA, Leiden, the Netherlands \\ email: tielens@strw.leidenuniv.nl
}

\begin{abstract}
Rather than a summary of the conference, I present here an overview of the status of the field and our progress over the last two decades from the points of view of astronomy, molecular physics, spectroscopy, and astrochemistry. While at first sight, progress may seem slow, actually, we have made an important stride forward. We have recognized now that the problem is very complex and identifying the carriers of the Diffuse Interstellar Bands will require a concerted effort of astronomers, molecular physicists, spectroscopists, and astrochemists. While this is a daunting prospect, we have identified the tools that we need to make this happen.
\end{abstract}

Keywords. ISM: molecules, Molecular spectroscopy, Astrochemistry

\section{Introduction}

Since the last DIB conference in 1994, we have witnessed a revolution in astronomy; a revolution that is shaking up our views of the Universe akin to the Copernican revolution some 450 years ago. We now know that planetary systems are bountiful: Essentially, every star has an associated planetary system and planets range from gas-rich super Jupiters, to gas-giant Neptunes, to super-Earth, to Earth-analog planets. Planets are truly widespread and diverse. While our understanding of the architecture of extrasolar planetary systems is still in its infancy and our measure of the inventory is greatly limited by selection effects, we have already started to ponder the conditions on these planets and their moons. New ground-based opportunities and space-based missions are actively being developed to pursue this aspect. In the end, this will lead us to address the biggest questions of them all; indeed the questions that, in my mind, define humanity: "What is our connection to the Universe ?" and "Are we alone?" As these questions have gotten more and more impetus over the last decade, the field of astrobiology has emerged from our great interest to understand the chemical evolution in the Universe from simple to complex molecules, especially those with biogenic potential and the roles they may play as primordial seeds in the origin of life on habitable worlds.

The study of the Diffuse Interstellar Bands is central to this quest of astronomers and astrochemists to trace the formation and development of chemical complexity in space. The DIBs are now well recognized as the signatures of complex molecules - complex by astronomical standards, not complex in the biological sense - and they herald a molecular Universe where molecules are widespread, diverse, and play an important role in the evolution and the physical and observational characteristics of the interstellar medium of galaxies (Tielens 2013). While sub-millimeter wavelengths provide a favorable tool for studying simple, linear molecules through their rotational transitions, this spectral region is not well suited to study molecular complexity in the ISM as the partition function greatly dilutes the brightness of spectral lines from large (non-linear) molecules. The ro-vibrational transitions in the mid-infrared have revealed the presence of $\sim 50 \mathrm{C}$ atom molecular species but specific identifications are difficult to make in this wavelength region as the subtle differences between individual spectra are difficult to disentangle if the molecular mixture consists of a variety of similar molecules. The electronic transitions 


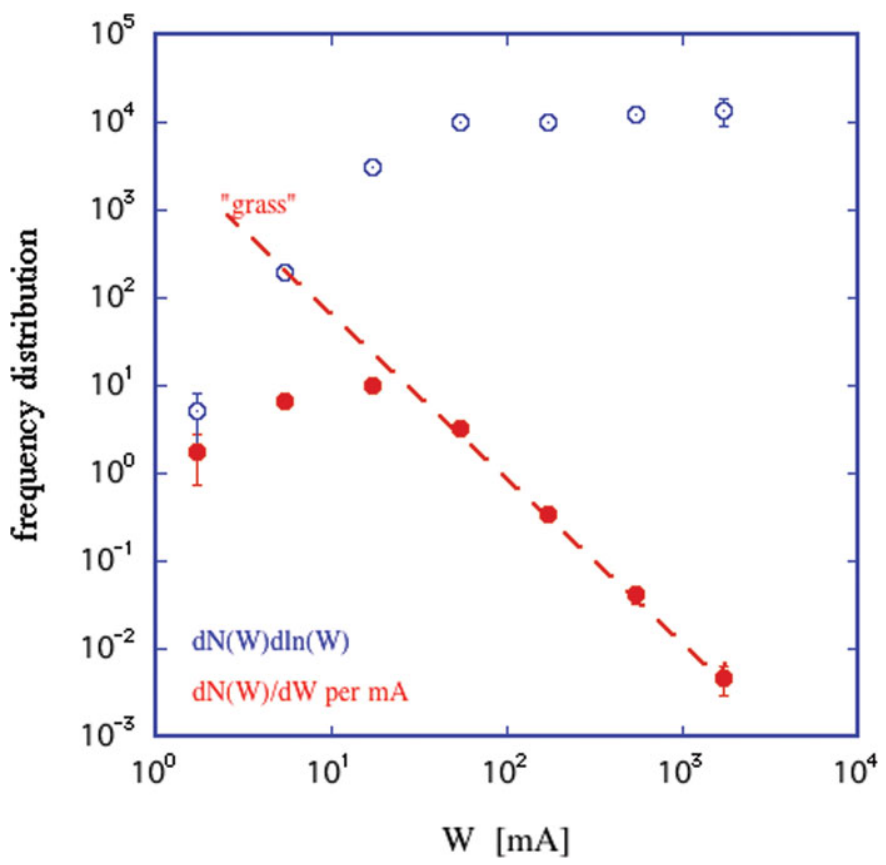

Figure 1. The frequency distribution function of DIBs with a given equivalent width $(W)$ in the wavelength range $3900-8100 \AA$ as measured towards the prototypical DIB-survey star, HD 183143, with $N_{H}=7.2 \times 10^{21} \mathrm{~cm}^{-2}$ (Hobbs et al. 2009). Red \& blue symbols refer to $d N / d W$ $\& W d N / d W$, respectively. The line indicates $N(\mathrm{DIBs}) \simeq 3 \times 10^{3} / E W(\mathrm{~m} \AA)$. The "grass" arrow indicates the spectral confusion limit. Incompleteness sets in around $30 \mathrm{~m} \AA$.

in the visible are molecule specific and hence might provide the preferred tool to study the inventory of complex molecules in the diffuse ISM of galaxies. However, this specificity is also a "curse" as conditions in the ISM are very different from terrestrial environments and hence the specific molecules involved are not necessarily in typical chemical catalogs and their excitation will be unlike that in laboratory environments. Hence, the molecule has to be "identified" before it can be studied spectroscopically in the laboratory to determine the fingerprints with which it can be identified. This catch 22 has stymied progress in the field of DIBs for decades. It is this challenge and this opportunity that we have to reconcile: Identification of the DIBs will require a complete understanding of the molecular Universe.

\section{Observations of DIBs}

As new technologies (e.g., CCDs) developed and new observing windows opened up (e.g., far-red and near-infrared), our knowledge of the DIB spectrum has slowly but steadily expanded over the last 20 years. In 1954, Herbig (1975) described 39 bands between 4400 and $6850 \AA$ as certainly or probably DIBs. In 1994, some 200 DIBs were catalogued by Jenniskens \& Desert (1994) while in 2009 the counter stood at some 400 DIBs (Hobbs et al. 2009). The strongest and best-known bands occur at 4430, 5778, $5780,5796,6177,6196,6284$, and $6614 \AA$. Most of the DIBs are, however, weak to very 


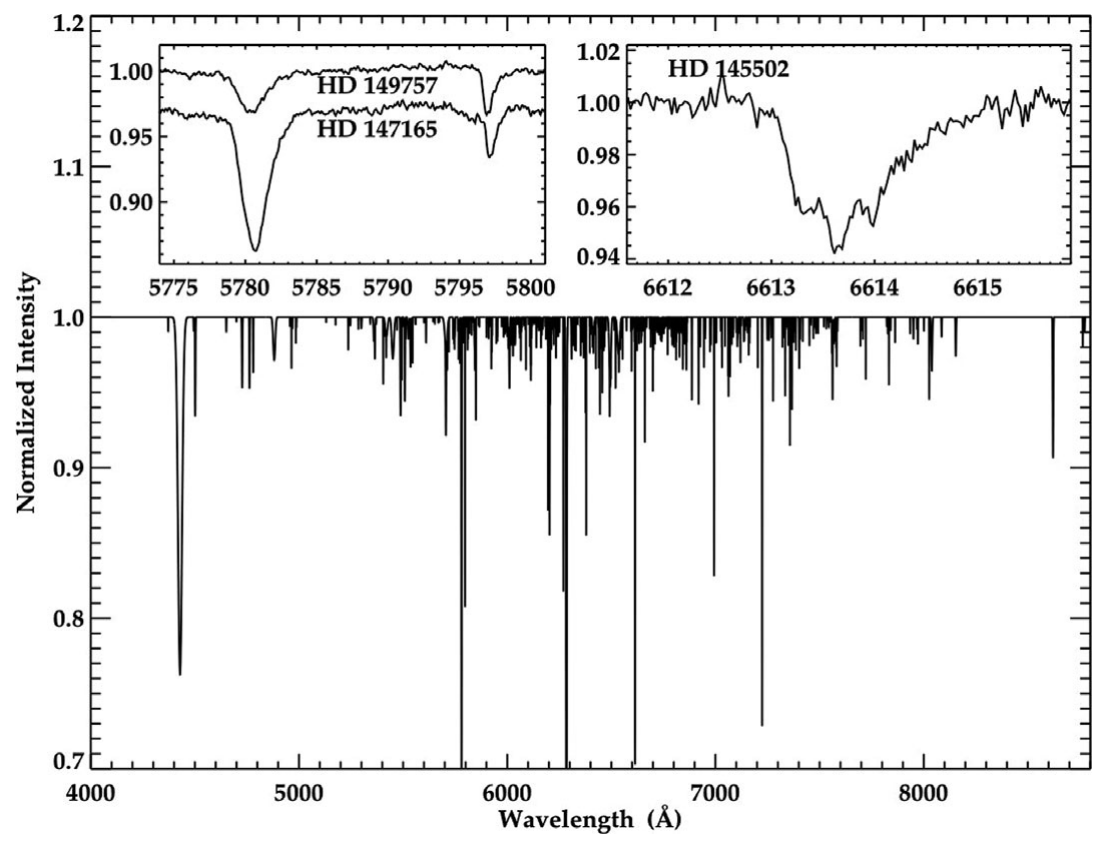

Figure 2. Some 400 Diffuse Interstellar Bands (DIBs) are present in visible absorption spectra of the interstellar medium. The synthesized spectrum of Diffuse Interstellar Bands within the 4300-6800 ^ wavelength range observed towards BD +63 1964 (Ehrenfreund et al. 1997) illustrates the great variety in relative strength and width of the DIBs. The right insert shows the detailed profile of the $6614 \AA$ DIB and its associated substructure observed towards the star HD 145502. The left insert illustrates the large variations in the strength of the DIB bands relative to each other for two well-studied DIBs (5780 and $5797 \AA$ ) on the basis of observations of the stars, HD 149757 and HD 147165. Figure courtesy of J. Cami.

weak $\left(W_{\lambda} / N_{H} \simeq 10^{-24} \AA \mathrm{H}\right.$-atom $\left.{ }^{-1}\right) \dagger$ but a subset of 30 - largely Herbig's original list have appreciable equivalent width $\left(W_{\lambda} / N_{H} \simeq 10^{-23} \AA \mathrm{H}^{-a_{0}}{ }^{-1}\right)$ and a few are strong $\left(W_{\lambda} / N_{H} \simeq 10^{-22} \AA\right.$ H-atom $\left.{ }^{-1}\right)$ (Fig. 1). Surveys are likely incomplete at the weak end and have not yet reached the spectral "confusion" limitf; Likely, there is a (very weak) DIB at every wavelength. Surveys are also incomplete for very broad DIBs. Broad DIBs - such as the $4430 \AA$ albeit much weaker - can be expected to be omnipresent $(\S 4)$. Typically, equal ranges in $W$ contribute equally to the integrated equivalent width of all DIBs. The DIB spectrum as known at the end of the 90ies is reproduced schematically in Figure 2.

Over the years, our insights in the correlation behavior of the DIBs has slowly shifted as detector technology advanced and more sensitive studies became possible: Initially, DIBs were largely considered as one "happy" family but in the early 90ies, it was discovered that correlations among DIBs were not perfect and the concept of different but related families was introduced where bands within one family correlate well together but not one-to-one with bands in other families (Chlewicki et al. 1987; Krelowski \& Walker 1987). By now it is clear that DIBs form really a "dysfunctional" family and no DIB correlates well with another one. Figure 2 illustrates this for the well-known DIBs, $\lambda \lambda 5780 \& 5797$ $\AA$. The best correlation is found for the pair 6614 and $6196 \AA$, but even for these two

$\dagger$ Strength (relative to the continuum) is expressed in terms of the equivalent width $W_{\lambda}=\int \frac{\left(F_{c}-F_{o}\right)}{F_{c}} d \lambda$ with $F_{c}$ and $F_{o}$ the continuum and observed flux.

$\ddagger$ The observed width of the DIBs varies from $\sim 0.5-30 \AA$ but is typically $0.7 \AA$. 
Table 1. Abundance of DIB carriers ${ }^{1}$

\begin{tabular}{|l|c|c|}
\hline $\begin{array}{l}\text { DIB } \\
\AA\end{array}$ & $\begin{array}{c}\text { Abundance } \\
\text { relative to } \mathrm{H}\end{array}$ & $f_{C}^{3,4}$ \\
\hline 4430 & $4 \times 10^{-9} / f$ & $10^{-3}$ \\
6284 & $7 \times 10^{-10} / f$ & $2 \times 10^{-4}$ \\
5780 & $4 \times 10^{-10} / f$ & $10^{-4}$ \\
$10^{-8} / f$ & $3 \times 10^{-3}$ \\
\hline
\end{tabular}

Notes:

${ }_{1}$ For HD 183143.

$2 f$ is the oscillator strength of the transition.

3 Fraction of the elemental carbon locked up in the carrier.

${ }^{4}$ Adopting an oscillator strength of $f=10^{-2}$ per C-atom.

DIBs the correlation is not truly perfect (Moutou et al. 1999; McCall et al. 2010). It should be mentioned that this has only been investigated well for DIBs with appreciable strength and whether this absence of correlation pertains to the weaker ones as well is to be determined.

Correlations with other tracers of the ISM have also been much pursued. There is a general (but not perfect) correlation with $\mathrm{HI}$ but the correlation with $\mathrm{H}_{2}$ is poorer. Indeed, the DIBs are generally weak in molecular clouds. Likewise, the strength of the DIBs correlates in a general sense with the dust column density. These correlation studies reveal global families that correlate with the behavior of the extinction curve; e.g., the 5797 is stronger in sight-lines with $\zeta$-Oph type extinction curves (pronounced $2175 \AA$ bump and strong FUV rise) while the 5780 correlates with $\sigma$-Sco extinction curves (weak $2175 \AA$ bump and weak rise of the FUV extinction). These family "divisions" are also sometimes reported as a relationship with the presence of simple diatomic molecules (e.g., $\mathrm{CO}, \mathrm{CH}, \mathrm{C}_{2}$ ) or as a dependence on the UV radiation field (Friedman et al. 2011; Sneden et al. 1991; Cami et al. 1997; Weselak et al. 2004) as all those aspects are, of course, loosely related. So, the $\sigma$-carriers may well trace gas in the atomic surfaces of diffuse clouds while the $\zeta$-carriers are related to their molecular cores.

In summary, from an observational point of view, each DIB is an " independentlyowned" franchise and the paradigm has developed "one-DIB-one-carrier", at least for the 20 strongest DIBs.

\section{Abundances of DIB carriers}

We can derive abundances of the carriers and these are summarized for the well-studied star, HD 183143, in table 1. Typical measured oscillator strengths for the first transition $\left(S_{0} \rightarrow S_{1}\right)$ of small PAHs and hydrocarbon chains are $0.01-0.1$ (Tuley et al. 1998; Maier et al. 2004; Gredel et al. 2011). As the size of these species increases, the effective number of electrons involved in the transition is expected to increase and the oscillator strength per $\mathrm{C}$ atom is roughly constant at $\sim 0.01$ (Watson 1994; Weisman et al. 2003). Thus, the carriers of the strong DIBs have to be very abundant (Table 1). Or they have to involve higher transitions (e.g., $S_{0} \rightarrow S_{2}$ ), which have larger oscillator strengths, but that is probably only true for the $4430 \AA$ band (see $\S 5$ ).

We can compare these abundances with molecular abundances measured through other means (Table 2). Not surprisingly, the abundance of carriers of these strong DIBs is comparable to that of simple diatomics measured in the optical. Rotational spectroscopy of small molecules in the (sub)-millimeter is, in general (but not always), a much more sensitive tool for detecting molecular species than visible electronic transitions and most species detected this way are - abundance-wise - not good candidates for the strong DIBs 
Table 2. Abundance of DIB carrier candidates

\begin{tabular}{|c|c|c|c|}
\hline Species & $\begin{array}{l}\text { Abundance } \\
\text { relative to } H\end{array}$ & $f_{C}^{1}$ & Method \\
\hline $\begin{array}{l}\mathrm{PAHs}_{\mathrm{s}} \\
\mathrm{C}_{60}{ }^{2} \\
\mathrm{CH} \\
\mathrm{C}_{2} \\
\mathrm{C}_{3} \mathrm{H}_{2} \\
\text { carbon chains }\end{array}$ & $\begin{aligned} & 10^{-7} \\
6 & \times 10^{-11} \\
5 & \times 10^{-8} \\
3 & \times 10^{-8} \\
& 10^{-9} \\
<<4 & \times 10^{-7} / \overline{N_{C}}\end{aligned}$ & $\begin{aligned} 2 & \times 10^{-1} \\
& 10^{-5} \\
& 10^{-4} \\
& 10^{-4} \\
3 & \times 10^{-6} \\
< & <10^{-3}\end{aligned}$ & $\begin{array}{c}\text { IR emission } \\
\text { IR emission } \\
\text { optical absorption } \\
\text { optical absorption } \\
\text { (sub)millimeter emission } \\
\text { infrared emission upper limit }\end{array}$ \\
\hline
\end{tabular}

Notes:

1 Fraction of the elemental carbon locked up in the carrier.

2 Higher abundances (factor 10) have been measured close to stars.

3 Abundance measured in NGC 7023 at $\simeq 35 "$ from the illuminating star. Higher abundances are measured close to the star (Berné \& Tielens 2012).

4 Upper limit on the integrated abundance of carbon chains determined from IR emission spectra. $\overline{N_{C}}$ is the average number of C-atoms per hydrocarbon chain.

(cf., Liszt, elsewhere in this volume). Likewise, fullerenes or their derivatives are unlikely candidates for the strong DIBs. As a class, PAHs are very abundant and they can account, in principle, for all the measured DIBs. Comparing abundances, any putative PAH carrier of the strong DIBs would need to correspond to $\sim 10^{-3}$ of the PAH population (see below). There are stringent upper limits on the abundance of hydrocarbon chains derived from the observed, interstellar IR emission spectrum (Tielens et al. 1999; Allamandola et al. 1999). Comparison of Tables 1 \& 2, suggests then that if responsible for the DIBs, the typical number of $\mathrm{C}$-atoms per hydrocarbon chain has to be very small. Moreover, as a class, small hydrocarbon chains (or fullerenes) cannot carry all of the DIBs. Of course, small hydrocarbon species, fullerenes, or less abundant PAHs are relevant candidates for transitions at the "grass" level and this aspect is now beginning to be explored and provides interesting limits on the abundances of specific molecules (Gredel et al. 2011; Salama et al. 2011). Typically these first studies have focused on small PAHs - as compared to interstellar IR emission standards - and derived abundance limits are very stringent $\left(f_{C} \simeq 10^{-6}\right)$.

The observed DIB characteristics imply that the interstellar molecular family contains a number of very abundant molecules that lock up some $0.01-0.1 \%$ of the elemental carbon (Table 1). These high abundances implies that the carriers of the strong DIBs are particularly stable and/or readily formed. The concept of a class of highly stable molecules has also been discussed in terms of the interstellar PAH family. The observed spectra of the many reflection nebulae are very similar, indeed to the smallest detail (Werner et al. 2009), and this has been taken to imply that under the most extreme conditions only the most stable species survive and will dominate the observed spectra - the so-called "grand-PAHs" (Tielens 2005). Inferred abundances of these PAHs are however much higher, typically $\sim 1 \%$.

\section{Spectroscopy of DIB carriers}

A number of research groups have made a dedicated effort to study relevant DIB carriers in the laboratory: notably John Maier and his group in Basel have focused on the spectroscopic characteristics of hydrocarbon chains, while Farid Salama and his group at NASA Ames, Huiskens and his group at Jena, and Thomas Pino and Philippe Brechignac at the IAS have put the emphasis on the PAH molecules (Maier et al. 2004, 2006; Salama et al. 2011; Gredel et al. 2011; Pino et al. 2011). These efforts are reviewed elsewhere in this volume. Here, I want to highlight two aspects: One, the oscillator strength of 


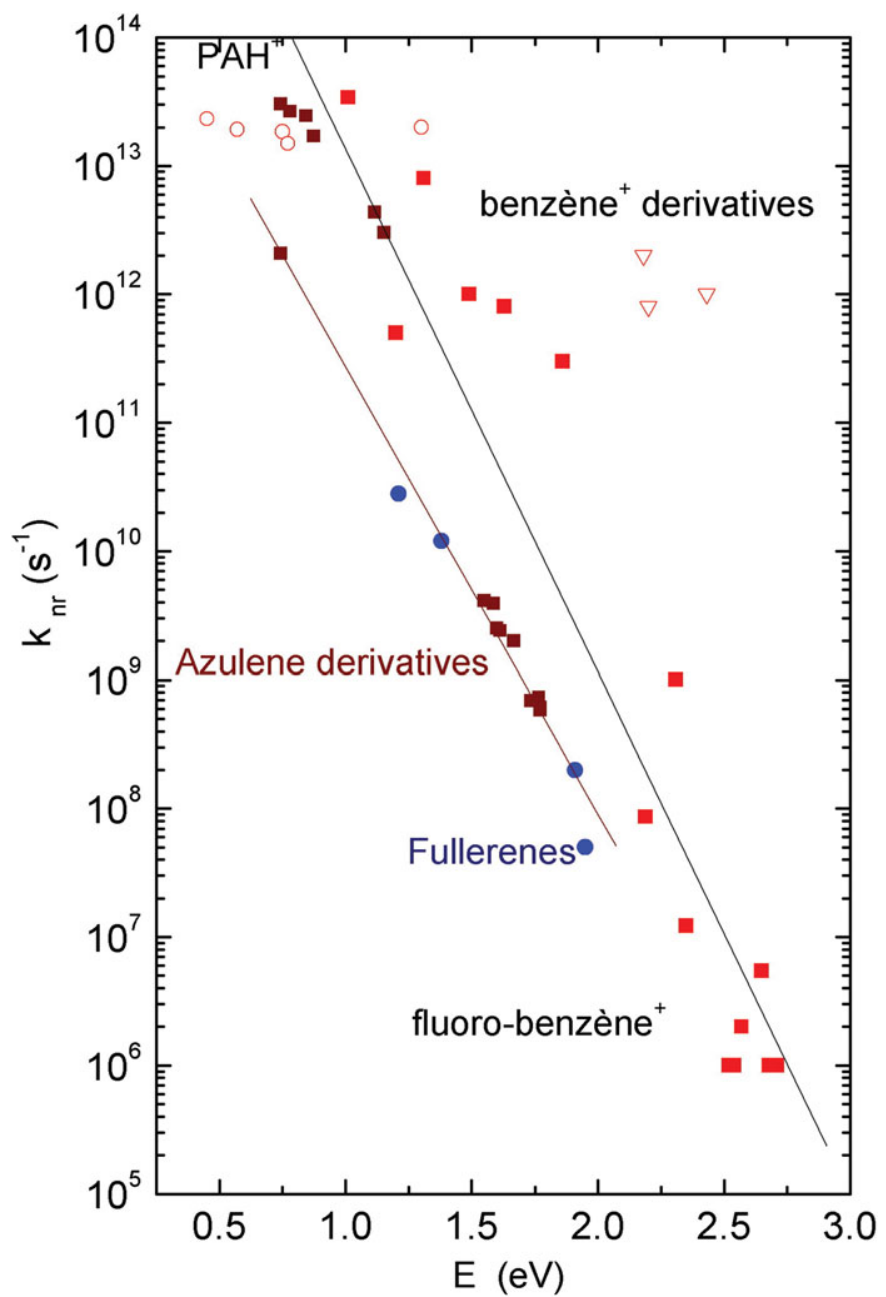

Figure 3. Nonradiative electronic relaxation rate of neutral and ionized PAHs and related compounds dominated by interconversion (Pino et al. 2011). The lines - guiding the eye signify the energy gap law. Figure kindly provided by Thomas Pino.

the first electronic transition is typically very weak (on a per C-atom basis) and hence high abundances are required to explain the strong DIBs $(\S 3)$. Higher transition are stronger but they are inherently very broad. And this line width issue is the second and important - aspect which should be highlighted. When the density of states becomes appreciable, the rate for internal conversion is very rapid and absorption bands are very broad. A transition dominated by intramolecular relaxation processes will have a Lorentz profile with a FWHM expressed in wavelength space, $\Delta \lambda$, given by (Leach 1987 ; Boudin 2000)

$$
\Delta \lambda \simeq 20\left(\frac{\lambda}{6000 \AA}\right)^{2}\left(\frac{k_{n r}}{10^{13} \mathrm{~s}^{-1}}\right) \quad \AA,
$$

where the non-radiative relaxation rate, $k_{n r}$, is controlled by an energy-gap law. Some relevant experimental data is summarized in Figure 3 (Pino et al. 2011), illustrating the energy gap law (derived from measured linewidths) but similar results also apply to hydrocarbon chains (Ding et al. 2003; Boguslavskiy et al. 2005; Boguslavskiy \& Maier 
2006). Thus, for visible transitions where the energy gap is $0.5-1 \mathrm{eV}$ (eg., typically one or more lower lying, excited electronic states), the line width will be some $20 \AA$. Hence, likely, only the $\lambda \lambda 4430 \AA$ band involves a transition from the ground-state to $n=2$ or higher. Conversely, most of the relevant transitions of carbon chains, PAHs, and fullerenes that we expect to be present are much broader than the typical DIB observed but interstellar spectra should be "riddled" with them. The "hunt" for such broad DIBs in interstellar spectra is however challenging.

\section{Molecular physics of DIB carriers}

In recent years, our understanding of the molecular physics involved in the DIB carriers has evolved considerably. In the past, it was generally assumed that the DIBs are due to electronic transitions of gas-phase molecules with low internal temperatures. Hence, they are in their lowest vibrational state with rotational temperature of $10-100 \mathrm{~K}$, depending on the molecule, but this view is now changing (Tielens 2013). Small radicals may indeed radiatively relax before further (reactive) collisions occur or the species is photo-dissociated or -ionized (Maier et al. 2011a). In contrast, the excitation of large PAHs will depend on the physical conditions - e.g., the radiation field - of the region (Joblin et al. 2002). Consider, UV pumping of PAHs will leave the species in a highly vibrationally excited state from which it will relax through IR emission (e.g., the IR emission features). Now, when the internal energy in the molecule drops below some critical value, $E_{c}$, the density of states will be so low that the modes will start to decouple (Kuzmin \& Stuchebrukhov 1985; Zelker \& Zewail 1983). That will leave the molecule vibrationally excited in the lowest vibrational modes and, without rapid Internal Vibrational Relaxation, energy in these modes can only be lost through radiation in these specific modes. For PAHs, the lowest (drumhead) mode occurs at $\simeq 59\left(50 / N_{c}\right) \mathrm{cm}^{-1}$ and has an integrated strength $\simeq 2.4 \times 10^{-19}\left(50 / N_{C}\right)^{4 / 5} \mathrm{~cm} /$ molecule (Boersma et al. 2011; Ricca et al. 2010). This corresponds to an Einstein A of $9.5 \times 10^{-4}\left(50 / N_{c}\right)^{14 / 5}$ $\mathrm{s}^{-1}$. For magnetic-dipole or electric-quadrupole transitions, the decay rates will be a factor $\sim(1 / 137)^{2} \simeq 5 \times 10^{-5}$ smaller. Consider specifically the circumcoronene molecule $\left(\mathrm{C}_{54} \mathrm{H}_{18}\right)$ with $1000 \mathrm{~cm}^{-1}$ of internal energy, the excitation temperature at decoupling will be $\sim 150 \mathrm{~K}$, several modes are accessible and will be appreciably populated, and the lowest mode at $40.6 \mathrm{~cm}^{-1}$ has no dipole-allowed transition. These deexcitation rates have to be compared to the UV pumping rates, $k_{u v} \simeq 4 \times 10^{-7}\left(N_{C} / 50\right) G_{0} \mathrm{~s}^{-1}$ and the collisional deexcitation rate, $n \gamma_{c o l} \simeq 10^{-9} n \mathrm{~s}^{-1}$ with $G_{0}$ the average interstellar (UV) radiation field in the Solar neighborhood and $n$ the density (Tielens 2005). With these values, levels with dipole-allowed transitions, will depopulate quickly (compared to the pumping rate). However, the population of IR-dark levels is set by the balance of UV pumping into and out of these states. The excitation of these modes will then be set by the (UV) Franck-Condon factors involved, the temperature of the radiation field, and the molecular excitation temperature at the time of decoupling. It will thus be sensitive to the local physical conditions (e.g., $G_{0}$ and the UV radiation field temperature which in turn depend on, for example, dust shielding).

This has a number of potential implications. The relationship between DIBs originating from the ground vibrational state and from IR-dark states depends on the Franck-Condon factors involved in the DIB transition. If the molecular geometry of the molecule changes appreciably between the ground and excited electronic state, this will shift transitions originating from dark states well clear from the ground state absorption. No perfect correlation is expected for such DIBs even though they originate from the same species. If the ground and excited electronic states have similar molecular geometries, then shifts 
may be quite small but would likely still affect the band profile, causing, perhaps, spectral substructure (cf., Fig. 2). This has also been examined as a cause of the observed variations in the profile of the DIB-related emission bands in the Red Rectangle (Sharp et al. 2006). Because the oscillator strength from IR-dark states may differ from that in the ground state, correlation studies would also be affected. The UV pumping process will dominate the rotational population (Rouan et al. 1992; Ysard \& Verstraete 2010). Hence, profiles of transitions controlled by rotations (rather than non-radiative processes) will be sensitive to the local conditions. While this discussion centers on PAHs, similar considerations pertain to other astrophysically relevant species (c.f., Oka, elsewhere in this volume). For example, the lowest vibration of pure carbon chains shifts through the 10 to $100 \mathrm{~cm}^{-1}$ range when the chain length increases from 4 to $10 \mathrm{C}$-atoms and for the larger carbon chains, deexcitation timescales may also approach the collisional, pumping, or chemical timescales. Finally, while this discussion does not affect the abundance considerations, it may imply that the interstellar complex molecule family is less diverse than the DIB correlation studies seem to imply.

From this discussion, it is clear that we will have to characterize much better the molecular physics of proposed carriers. I would say, invariably, the excitation conditions in the laboratory differ greatly from those in space. Hence, among others, we will need to quantify the role of UV pumping and collisional de-excitation in setting the rotational population of putative candidates and we will have to characterize low lying vibrational states and the Franck-Condon factors involved in relevant absorption processes.

\section{Chemistry of DIB carriers}

There are two main ideas on the chemistry involved in the DIB carriers, basically, related to the characteristic of the molecules involved. First, the carriers might be small hydrocarbon species that are formed through ion-molecule or neutral radical-radical reactions from small precursors and some attention has been given to the chemistry of large interstellar hydrocarbon chains (Thaddeus 1995; Bettens \& Herbst 1997). However, such models face severe challenges as the abundances are expected to drop very rapidly with chain size. As the observed abundances of small hydrocarbon species is about the abundance required to explain the strong DIBs (Table $1 \& 2$ ), this strongly argues against this chemical pathway. Indeed, models based upon bottom-up chemistry typically produce abundances of order $\sim 10^{-11}$ for hydrocarbon chains with $\simeq 10 \mathrm{C}$ atoms (Bettens \& Herbst 1997) often considered as likely DIB candidates. In a way, this is a restatement of the argument that small hydrocarbon chains and radicals observed at mm-wavelength are not abundant enough to be viable DIB candidates (cf., Liszt as well as Maier elsewhere in this volume). Essentially, in the diffuse ISM, hydrocarbons smaller than some $30 \mathrm{C}$-atoms are readily photodissociated by the strong UV radiation field and a rapid reformation process is required to keep their abundances up. Perhaps, the nonequilibrium conditions associated with turbulent diffusion layers (e.g., warm, relatively dense gas temporarily compressed and heated by local dissipation of turbulence) may be conducive to produce higher abundances of relevant species.

The second route is appropriate for large PAHs and fullerenes. These species are generally assumed to be very stable under interstellar conditions and they are thought to form at high temperatures and densities in stellar ejecta in a process akin to PAH and dust formation in sooting flames. Detailed models have been developed for this process (Frenklach \& Feigelson 1989; Cherchneff et al. 1992; Cherchneff 2012). These large species are then assumed to survive for a very long time in the ISM. These models, too, face issues as recent studies calculate lifetimes of PAHs against destruction in in- 


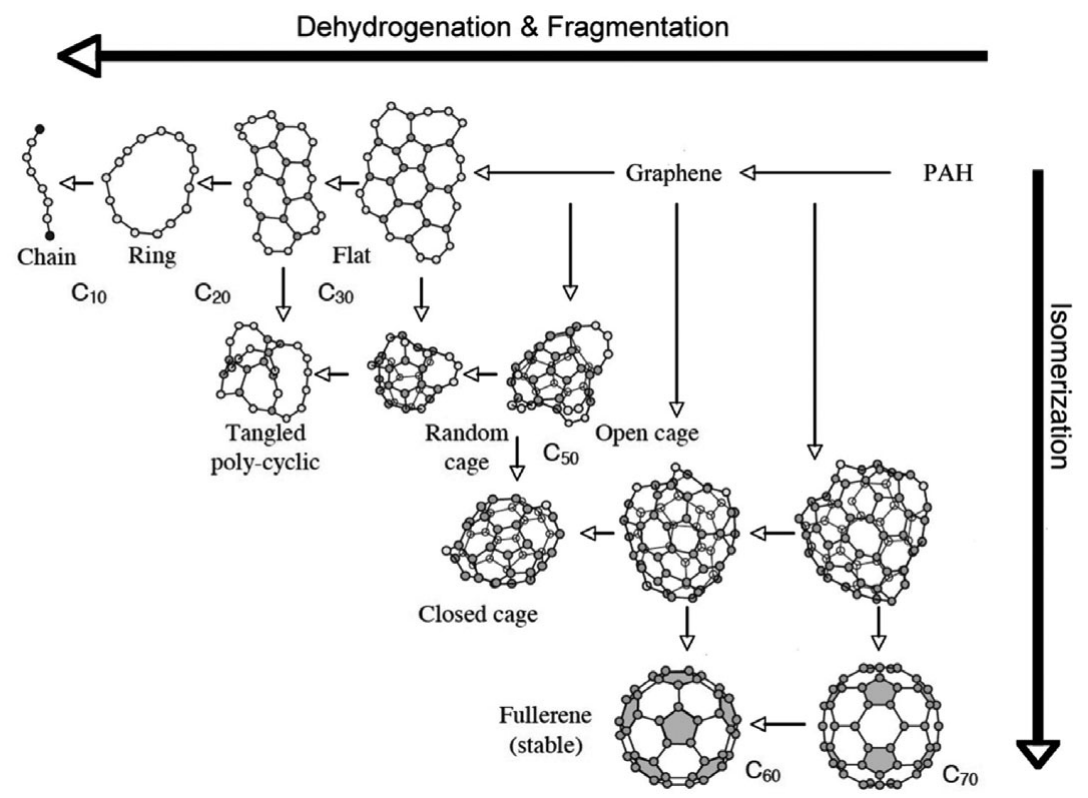

Figure 4. Schematic representation of top-down interstellar carbon chemistry. The chemical evolution of PAHs in the interstellar medium under the influence of UV photons combines the effects of dehydrogenation and fragmentation with those of isomerization. Fully hydrogenated PAHs - injected by stars into the ISM - are at the top right side. Near bright stars, UV photolysis will preferentially lead to complete H-loss (e.g., the "weakest link") and the formation of graphene. Further fragmentation may lead to the formation of flats, rings, and chains. However, this process competes with isomerization to various types of stable intermediaries such as cages and fullerenes.

terstellar shocks of only some $100 \mathrm{Myr}$ (Micelotta et al. 2010a) much less than the PAH injection timescale $(\simeq 2$ Byr). To compensate for this rapid destruction, PAHs may have to be "formed" non-chemically in-situ in the ISM through, for example, shattering by grain-grain collisions in shocks (Jones et al. 1996).

Recently, a third chemical route has been identified - "top-down" interstellar chemistry - where large interstellar molecules are broken down into smaller species (Fig. 4; Berné \& Tielens (2012)). In this model - driven by the observation that the PAH abundance decreases rapidly while the $\mathrm{C}_{60}$ abundance increases close to star, HD 200775, powering the reflection nebula, NGC 7023 - UV photons first strip interstellar PAHs from their $\mathrm{H}$ atoms to form graphene sheets (Ekern et al. 1997) and this graphene is subsequently broken down to smaller hydrocarbons (Fig. 4). Along the way, some of the graphene may isomerize to cages and fullerenes. In this trickle-down chemistry, large species (formed elsewhere under dense warm conditions) are the precursors of small species in UV rich environments such as photodissociation regions and the diffuse ISM (Pety et al. 2005). This route may well be a source of large hydrocarbon chain species that are difficult to build up from the bottom.

The complexity of the chemistry of interstellar gas is only now realized and these three different basic routes - as well as mixed variants - will have to be explored more fully. This will require dedicated laboratory studies on the relevant reaction rates. 


\section{Identification of DIB carriers}

Identification of the carriers of the DIBs has turned out to be very challenging as the list of potential candidates is very large and laboratory studies are very demanding. Among the leading candidates are hydrocarbon chains (Maier et al. 2004; Fulare et al. 1993a), PAHs (Bréchignac \& Pino 1999; Biennier et al. 2003; Kokkin et al. 2008), and fullerenes (Foing \& Ehrenfreund 1994; Sassara et al. 2001), largely because there is independent evidence (c.f., Table 2) for the presence of these species in the ISM (and that is one way to limit the scope of the search). Electronic transitions in the visible require either open shell radicals, cations or anions, or very large species. Because these species are reactive or unstable in laboratory settings or are not very volatile, special techniques have to be used (for a short review of these techniques, see Pino et al. (2011)). Many laboratory studies have employed matrix isolation techniques but the matrix will shift and broaden the bands and, while this can provide tantalizing suggestions, identification requires further proof. A number of criteria can be formulated before an identification will be widely accepted by the community, including an exact match of peak positions, profiles, and relative strength of (at least) two DIBs with laboratory gas phase spectra (Maier 1994). But, as has recently been realized, IR dark states (see above) can have appreciable populations. Likewise, rotational populations may vary readily as the excitation conditions change due to variations in the pumping UV field and or density. The implications of these are now only beginning to be investigated and one or all of these criteria may have to be relaxed somewhat, as has been illustrated for rotational excitation variations by Oka (elsewhere in these proceedings) as well as for the profile variations of the Red Rectangle emission bands (Sharp et al. 2006).

While a few specific species have been put forward as candidates for particular DIBs, invariably, these have fallen by the wayside if tested according to these strict criteria. Specifically, the coincidence of 5 of the 7 vibronic bands in the lowest energy transition of the anion, $\mathrm{C}_{7}^{-}$reinvigorated the field when it was announced in 1998 (Tuley et al. 1998). However, later astronomical studies casted serious doubt on this identification since the observed peak position and width of the strongest (origin) band of this species (in fact, all weak vibronic bands as well) do not agree with calculated profiles (McCall et al. 2001). The assignment of the 9577 and $9632 \AA$ bands to $\mathrm{C}_{60}^{+}$(Foing \& Ehrenfreund 1994) faces issues that were never fully resolved (Maier 1994) as the identification was based upon matrix-isolation studies (Fulare et al. 1993b) and peak positions will shift due to matrix interaction. Furthermore, in the laboratory studies, the relative strength of the two bands varies depending on the experimental conditions and this has been attributed to the presence of two slightly different, distorted structures of $\mathrm{C}_{60}^{+}$trapped in the matrix (Fulare et al. 1993b). So, this proposed identification still has to await laboratory (gas phase) confirmation. The origin band of the linear propadienylidene anion, $l-\mathrm{C}_{3} \mathrm{H}_{2}^{-}$, at $6994 \AA$ occurs close to a weak DIB (Güthe et al. 2001), but there is no exact match in a comparison with high resolution laboratory spectra (McCall et al. 2002). Following a suggestion by Linnartz et al. (2010), the broad DIBs at 4881 \& $5450 \AA$ have been attributed to vibronic absorptions by the radical, propadienylidene $\left(l-\mathrm{C}_{3} \mathrm{H}_{2}\right)$ based on the excellent agreement in peak position and width (Maier et al. 2011a). Subsequent studies revealed intensity variations between the astronomical bands, calling this identification into question (Krełowski et al. 2011). A weak DIB at $5069 \AA$ has been attributed to the gas phase origins transition of $\mathrm{HC}_{4} \mathrm{H}^{+}$(Krełowski et al. 2010). However, accurate profile calculations revealed differences in peak position and width with the observed DIB and this assignment, too, may bite the dust (Maier et al. 2011b). Of course, these assessments may have to be revisited as our understanding of the molecular physics involved has 
improved; e.g., the role of IR dark states and rotational excitation differences between laboratory studies and the ISM $(\S 5)$.

\section{The way forward}

At the time of the $1^{\text {st }}$ conference on the Diffuse Interstellar Bands in 1994, the mood was very much upbeat. The recent suggestion of $\mathrm{C}_{7}^{-}$and $\mathrm{C}_{60}^{+}$as $\mathrm{DIB}$ carriers had invigorated the field and, even if these were not everyone's favorite candidate, the general feeling was "surely we would lick the problem soon!". In the intervening years, this promise never really materialized and indeed generally accepted identifications have remained as elusive as ever. The general feeling at this conference is that in many ways we seem to be farther from our goal. Indeed, despair seems to have settled in and - as actually has been true for most of the last century - studies of the DIBs has become sort of an hobby which can be indulged in every once and a while but is not an acceptable career move for a young scientist. This feeling is too bleak. In my opinion, we have made important progress and the solution to the age-old DIB question is "in-hands".

In this regard, the most important step forward has been that we have realized the enormous complexity of the issue of the DIB carriers; much more complex than a single scientist can solve by himself. Solving the DIB problem will require the close cooperation of astronomers, molecular physicists, astrochemists, and spectroscopists - each contributing their pieces of the puzzle. Spectroscopists will have to study the visible spectroscopic signatures of relevant carriers as well as the general photophysics of these species. In order to keep the molecular zoo to be studied tractable, these studies will have to be guided by astrochemists who can identify those species that might be particularly relevant. Their models will have to explore the inherent non-steady-state conditions of the interstellar medium, including aspects of the global cycling associated with star-death and star-formation as well as the transient chemistry of, for example, turbulent dissipation regions. Laboratory studies of key chemical rates will have to be measured in the laboratory in order to make these astrochemical studies realistic. As the excitation conditions in laboratory settings will differ from those in the ISM, molecular physicists in close collaboration with astrophysicists will have to develop models for the excitation processes relevant for the interstellar environment and evaluate the line profiles based on a deep understanding of the photophysics and using the molecular constants measured in the laboratory. Astronomers will have to play a key role in guiding these studies, evaluating the results, and comparing calculated and measured spectra.

This is a long term program. As no single scientist, no single institute, no single country can be expected to solve the DIBs by itself, the DIB community will have to organize itself to make this happen. I envision an "open" international consortium with a clear definition of overall goals and near-term objectives which can be reached through joint projects involving the different disciplines. Key words are shared projects which make use of shared facilities, shared infrastructure and "shared" students and staff. Progress will require free and open exchange of data and direct communication channels. Openaccess, web-based data bases - through the VAMDC - can play an important role in this. Communication can also be effectuated through a newsletter appearing a few times a year. It will be very important for the community to meet regularly in workshops (a conference every other decade is not enough!) and COST actions could be explored for this. Equally important, the community should organize summer schools where the future generation of researchers will be trained in relevant aspects of the disciplines involved. Such an effort will require funding and, while long term stable funding has remained elusive in a European context, the Marie Curie Initial Training Network program under Horizons 
2020 can provide an initial avenue for community organization. It is my expectation that, with such a concerted program, we can indeed expect to cash in on the promise of the DIBs and to identify the full extent of the molecular Universe.

\section{References}

Allamandola, L. J., Hudgins, D. M., Bauschlicher, C. W., Jr., \& Langhoff, S. R. 1999, A\& A, 352,659

Berné, O. \& Tielens, A. G. G. M. 2012, Proceedings of the National Academy of Science, 109, 401

Bettens, R. P. A. \& Herbst, E. 1997, ApJ, 478, 585

Biennier, L., Salama, F., Allamandola, L., \& Scherer, J. 2003, J. Chem. Phys., 118, 7863

Boersma, C., Bauschlicher, C. W., Jr., Ricca, A., et al. 2011, ApJ, 729, 64

Boguslavskiy, A. E., Ding, H., \& Maier, J. P. 2005, JCP, 123, 034305

Boguslavskiy, A. E. \& Maier, J. P. 2006, JCP, 125, 094308

Boudin, N., 2000, PhD thesis, Université Paris-Sud

Bréchignac, P. \& Pino, T. 1999, A\&SA, 343, L49

Cami, J., Sonnentrucker, P., Ehrenfreund, P., \& Foing, B. H. 1997, A\&A A, 326, 822

Cherchneff, I., Barker, J. R., \& Tielens, A. G. G. M. 1992, ApJ, 401, 269

Cherchneff, I. 2012, A\&BA, 545, A12

Chlewicki, G., de Groot, M. S., van der Zwet, G. P. et al. 1987, A\&A, 173, 131

Ding, H. et al. 2003, JCP, 119, 814

Ehrenfreund, P., Cami, J., Dartois, E., \& Foing, B. H. 1997, A\&\&A, 317, L28

Ekern, S. P., Marshall, A. G., Szczepanski, J., \& Vala, M. 1997, ApJ, 488, L39

Foing, B. H. \& Ehrenfreund, P. 1994, Nature, 369, 296

Frenklach, M. \& Feigelson, E. D. 1989, ApJ, 341, 372

Friedman, S. D., York, D. G., McCall, B. J., et al. 2011, ApJ, 727, 33

Fulare, J., Lessen, D., Freivogel, P., \& Maier, J. P. 1993, Nature, 366, 439

Fulare, J., Jacobi,M. \& Maier, J. P. 1993, Chem Phys Lett., 211, 227

Gredel, R., Carpentier, Y., Rouillé, G., et al. 2011, A\&\&A, 530, A26

Güthe, F., Tulej, M., Pachkov, M. V., \& Maier, J. P. 2001, ApJ, 555, 466

Herbig, G. H. 1975, ApJ, 196, 129

Hobbs, L. M., York, D. G., Thorburn, J. A., et al. 2009, ApJ, 705, 32

Jenniskens, P. \& Desert, F.-X. 1994, A\& A Suppl, 106, 39

Joblin, C., Toublanc, D., Boissel, P., \& Tielens, A. G. G. M. 2002, Molecular Physics, 100, 3595

Jones, A. P., Tielens, A. G. G. M., \& Hollenbach, D. J. 1996, ApJ, 469, 740

Kokkin, D. L., Troy, T. P., Nakajima, M., et al. 2008, ApJ, 681, L49

Krelowski, J. \& Walker, G. A. H. 1987, ApJ, 312, 860

Krełowski, J., Beletsky, Y., Galazutdinov, G. A., et al. 2010, ApJ, 714, L64

Krełowski, J., Galazutdinov, G., \& Kołos, R. 2011, ApJ, 735, 124

Kuzmin, M. V. \& Stuchebrukhov, A. A. 1985, Chem Phys Lett., 119, 556

Leach, S. 1987, in "Polycyclic Aromatic Hydrocarbons and Astrophysics", eds. A. Léger, L. d'Hendecourt, N. Boccara, (Reidel; Dordrecht), p99

Linnartz, H., Wehres, N., van Winckel, H., et al. 2010, A\& A, 511, L3

Maier, J. P. 1994, Nature, 370, 423

Maier, J. P.,, Walker, G. A. H., \& Bohlender, D. A. 2004, ApJ, 602, 286

Maier, J. P., Boguslavskiy, A. E., Ding, H., Walker, G. A. H., \& Bohlender, D. A. 2006, ApJ, 640, 369

Maier, J. P., Walker, G. A. H., Bohlender, D. A., et al. 2011, ApJ, 726, 41

Maier, J. P., Chakrabarty, S., Mazzotti, F. J., et al. 2011, ApJ, 729, L20

McCall, B. J., Thorburn, J., Hobbs, L. M., Oka, T., \& York, D. G. 2001, ApJ, 559, L49

McCall, B. J., Oka, T., Thorburn, J., Hobbs, L. M., \& York, D. G. 2002, ApJ, 567, L145

McCall, B. J., Drosback, M. M., Thorburn, J. A., et al. 2010, ApJ, 708, 1628

Micelotta, E. R., Jones, A. P., \& Tielens, A. G. G. M. 2010, A\&̋A, 510, A36 
Moutou, C., Krełowski, J., D’Hendecourt, L., \& Jamroszczak, J. 1999, A\& A, 351, 680

Pety, J., Teyssier, D., Fossé, D., Gerin, M., Roueff, E., Abergel, A., Habart, E., \& Cernicharo, J. 2005, A\& $A, 435,885$

Pino, T., Carpentier, Y., Féraud, G., et al. 2011, EAS Publications Series, 46, 355

Ricca, A., Bauschlicher, C. W., Jr., Mattioda, A. L., Boersma, C., \& Allamandola, L. J. 2010, ApJ, 709, 42

Rouan, D., Leger, A., Omont, A., \& Giard, M. 1992, A\&3 A, 253, 498

Salama, F., Galazutdinov, G. A., Krełowski, J., et al. 2011, ApJ, 728, 154

Sassara, A., Zerza, G., Chergui, M., \& Leach, S. 2001, ApJS, 135, 263

Sharp, R. G., Reilly, N. J., Kable, S. H., \& Schmidt, T. W. 2006, ApJ, 639, 194

Sneden, C., Woszczyk, A., \& Krelowski, J. 1991, PASP, 103, 1005

Thaddeus, P. 1995, The Diffuse Interstellar Bands, 202, 369

Tielens, A. G. G. M., Hony, S., van Kerckhoven, C., \& Peeters, E. 1999, The Universe as Seen by ISO, 427,579

Tielens, A. G. G. M. 2005, The Physics and Chemistry of the Interstellar Medium, (Cambridge, UK: Cambridge University Press)

Tielens, A. G. G. M. 2008, ARA\&A, 46, 289

Tielens, A. G. G. M. 2013, Rev Mod Phys, 85, 1021

Tuley, M., Kirkwood, D. A., Pachkov, M., \& Maier, J. P. 1998, ApJ, 506, L69

Watson, J. K. G. 1994, ApJ, 437, 678

Weisman, J. L., Lee, T. J., Salama, F., \& Head-Gordon, M. 2003, ApJ, 587, 256

Werner, M. W., Sellgren, K., \& Livingston, J. 2009, BAAS, 41, \#412.15

Weselak, T., Galazutdinov, G. A., Musaev, F. A., \& Krełowski, J. 2004, A\&A, 414, 949

Ysard, N. \& Verstraete, L. 2010, A\&A, 509, A12

Zelker, P. M. \& Zewail, A. H. 1983, Chem Phys Lett, 102, 113 